\title{
The Italian Society for Rheumatology clinical practice guidelines for the diagnosis and management of knee, hip and hand osteoarthritis
}

\author{
A. Ariani ${ }^{1,2}$, M. Manara ${ }^{1,3}$, A. Fioravanti ${ }^{4}$, F. Iannone ${ }^{5}$, F. Salaffi ${ }^{6}$, N. Ughi $i^{1,3}$, \\ I. Prevete ${ }^{1,7}$, A. Bortoluzzi ${ }^{1,8}$, S. Parisi ${ }^{1,9}$, C.A. Scirè ${ }^{1,8}$ \\ ${ }^{1}$ Epidemiology Research Unit, Italian Society for Rheumatology (SIR), Milan, Italy; ${ }^{2}$ Department of Medicine, \\ Internal Medicine and Rheumatology Unit, University Hospital of Parma, Italy; ${ }^{3}$ Rheumatology Clinic, \\ Centro Specialistico Ortopedico-Traumatologico Gaetano Pini-CTO ASST Gaetano Pini, Milan, Italy; \\ ${ }^{4}$ Rheumatology Unit-Azienda Ospedaliera Universitaria Senese, Siena, Italy; ${ }^{5}$ Department of Emergency \\ and Organ Transplantation, Rheumatology Unit, University of Bari, Italy; ${ }^{6}$ Rheumatology Clinic, Ospedale \\ Carlo Urbani, Università Politecnica delle Marche, Jesi (AN), Italy; ${ }^{7}$ Rheumatology Unit, Azienda Ospedaliera \\ San Camillo-Forlanini, Rome, Italy; ${ }^{8}$ Rheumatology Section, Department of Medical Sciences, University of \\ Ferrara, Italy; ${ }^{9}$ Rheumatology Unit, Azienda Ospedaliera Città della Salute e della Scienza di Torino, Turin, Italy
}

\section{SUMMARY}

Osteoarthritis (OA) is the most common musculoskeletal disease leading to functional decline and loss in quality of life. Knees, hands and hips are frequently affected joints with a relevant clinical and socio-economic burden. An evidence-based approach to OA management is essential in order to improve patients' health and to decrease social burdens. Since new international clinical practice guidelines (CPGs) focused on diagnosis or pharmacological/non-pharmacological treatment have become available in the last ten years, the Italian Society for Rheumatology (SIR) was prompted to revise and customize them for a multidisciplinary audience of specialists involved in the management of OA.

The framework of the Guidelines International Network Adaptation Working Group was adopted to identify, appraise (AGREE II), synthesize, and customize the existing CPGs on OA to the needs of the Italian healthcare context. The task force, consisting of rheumatologists from the SIR epidemiology research unit and a committee with experience of OA, identified key health questions to guide a systematic review of published guidelines. The target audience included physicians and health professionals who manage OA. An external panel of stakeholders rated the guidelines.

From a systematic search in databases (Pubmed/Medline, Embase) and grey literature, 11 CPGs were selected and appraised by two independent raters. Combining evidence and statements from these CPGs and clinical expertise, 16 guidelines were developed and graded according to the level of evidence. Agreement and potential impact on clinical practice were assessed.

These revised guidelines are intended to provide guidance for diagnosis and treatment of OA and to disseminate best evidence-based strategies management of the disease.

Key words: Clinical practice guidelines; recommendations; osteoarthritis; diagnosis, treatment.

Reumatismo, 2019; 71 (S1): 5-21

\section{INTRODUCTION}

steoarthritis (OA) is the most common musculoskeletal disease, associated with a relevant decrease in quality of life (1). Its prevalence, currently around $20-30 \%$ of the population of the wealthier countries, will increase further with the progressive lengthening of life expectancy $(1,2)$. After cardiovascular diseases, $\mathrm{OA}$ is the most frequent cause of disability and reduction in work activity (1). Moreover, $\mathrm{OA}$ is associated with other chronic conditions (e.g. obesity, arterial hypertension, diabetes mellitus). OA itself is often a factor limiting the prevention and treatment of cardiovascular and metabolic disorders (3). The economic burden is relevant both for indirect (e.g. loss of working days, worsening of general conditions, need for assis-
Corresponding author: Alarico Ariani Department of Medicine, Internal Medicine and Rheumatology Unit, Azienda Ospedaliero Universitaria di Parma Via Gramsci, 14 - 43100 Parma, Italy E-mail: aariani@ao.pr.it 
tance) and direct costs. Misuse of laboratory and instrumental exams, inadequate access to specialists' care and ineffective treatment (pharmacological or not) lead to an increase in healthcare expenditure (4). Therefore, the drafting, dissemination and application of clinical practice guidelines (CPGs) for the management of knee, hip and hand OA has a pivotal role in optimizing the health care system.

The Italian Society for Rheumatology (SIR) undertook to address this complex situation by issuing guidelines on the management of knee, hip and hand OA respectively in 2004, 2006 and 2013 (5-7). Since their publication, new scientific evidence concerning OA therapy and diagnosis has emerged with a potential impact on daily clinical practice. The most recent CPGs have been developed by supranational scientific societies or countries with health systems that are different from the Italian one. Simple translation of those recommendations into our national context may not be advisable.

Updating high-quality recommendations and GCPs in clinical practice requires substantial consumption of time and resources. Investments are also needed to promote their applicability in local contexts. The ADAPTE methodology, based on already existing guidelines, is a valid alternative to de novo local GCP development $(8,9)$.

\section{Objective}

This guideline aims to offer revised, evidence-based, and adapted guidelines for the diagnosis and treatment of patients with knee, hip and hand OA (both primary and secondary) in Italy.

\section{Target patient population}

Adult patients (age $\geq 18$ years) with suspected or established knee, hip and/or hand OA.

\section{Target users}

Attending physicians (rheumatologists, physiatrists, geriatricians, internists, orthopedic surgeons and general practitioners) and health professionals who manage patients with knee, hip and hand $\mathrm{OA}$ in primary care and hospital, community and academic practice settings; patients, policy makers and those responsible for commissioning care for patients with knee, hip and hand OA in the Italian National Health Service (NHS).

\section{What is covered}

These guidelines are focused on the different phases of the management of patients with OA, including diagnosis, pharmacological and non-pharmacological treatment. These phases are specified by clinical questions of interest identified a priori. The guidelines are subsequently developed on the basis of these. The joints taken in to account are the knee, hip and hand.

\section{Areas that are not covered}

Recommendations on surgical management and post-operative rehabilitation of patients with OA are not included in this guideline.

\section{Funding}

These guidelines matched no specific funding from any bodies in the public, commercial sectors or non-profit organization. Non-economic support, such as meetingrooms and secretariat services, was provided by SIR to help carry out the work described in this manuscript.

\section{MATERIALS AND METHODS}

\section{Approach to guideline development}

A systematic approach based on the framework of the Guidelines International Network Adaptation Working Group (http:// www.g-i-n.net), following the work of the ADAPTE collaboration $(8,9)$, was adopted to identify, appraise, synthesize, and customize the existing international guidelines to the needs of the Italian healthcare context.

\section{Assembly of the Working Group}

The Working Group consisted of ten rheumatologists on behalf of the SIR. Six rheumatologists (AA, MM, NU, IP, AB, SP), and a project coordinator (CS) from the SIR epidemiology research unit were re- 
sponsible for CPG methodology and development. Three experts in OA management were appointed by SIR (AF, FI, FS) and included in each phase of the guideline development by attending a working group at the $54^{\text {th }}$ SIR National Meeting, contributing to e-mail discussions and participating in a web-meeting.

\section{Stakeholder involvement}

The draft of these guidelines has been revised and rated by an external multidisciplinary commission composed of seven rheumatologists (including an opinion leader in OA as author of the guidelines), one general practitioner, one physiatrist, one orthopedic surgeon, one health professional, and one representative of patients' associations at the invitation of SIR. The guidelines were developed without any input from, or cooperation with, any pharmaceutical company.

\section{Defining the scope}

The scope of the revised guidelines and a comprehensive list of potential issues on OA management that needed to be addressed was developed a priori by the working group and agreed by consensus. Sixteen questions were identified (Table I) and guided the focused systematic literature searches.

Table I - Key questions regarding the overall management of patients with osteoarthritis. Sixteen health questions guided the systematic review and the adapted guidelines development.

\begin{tabular}{|c|c|c|}
\hline No. & Text of the health question & No. recommendation \\
\hline \multicolumn{3}{|c|}{ DIAGNOSIS } \\
\hline 1. & Which are the typical symptoms, signs and anamnestic characteristics (e.g., OA familiarity) of OA? & 1 \\
\hline 2. & When is imaging useful? & II \\
\hline 3. & Which is the first line imaging exam? & III \\
\hline 4. & When should the synovial fluid exam be performed? & IV \\
\hline 5. & Which laboratory tests should be performed? & V \\
\hline \multicolumn{3}{|c|}{ OVERARCHING TREATMENT PRINCIPLES } \\
\hline 6. & $\begin{array}{l}\text { Does optimal management of OA require a combination of pharmacological and non-pharmacological } \\
\text { treatments? }\end{array}$ & $\mathrm{VI}$ \\
\hline 7. & According to which factors should $\mathrm{OA}$ treatment be individualized? & VII \\
\hline \multicolumn{3}{|c|}{ ANALGESIC TREATMENT } \\
\hline 8. & $\begin{array}{l}\text { Which analgesic should be preferred for the first line treatment? And what should be done } \\
\text { in the case of its ineffectiveness? }\end{array}$ & VIII \\
\hline 9. & When and for how long should the NSAIDs be taken? & IX \\
\hline 10. & Which topical pharmacological treatments should be used? & $\mathrm{x}$ \\
\hline \multicolumn{3}{|c|}{ PHARMACOLOGIC TREATMENT } \\
\hline 11. & $\begin{array}{l}\text { Which are the indications for intra-articular infiltrations of hyaluronic acid, steroids } \\
\text { or mesenchymal stem cells/platelet-rich plasma? }\end{array}$ & $X I$ \\
\hline 12. & What are the indications for oral therapy with SYSADOA (chondroprotectors)? & XII \\
\hline \multicolumn{3}{|c|}{ NON PHARMACOLOGIC TREATMENT } \\
\hline 13. & Are patient education, lifestyle changes and therapeutic exercise useful? & XIII \\
\hline 14. & What is the utility (from the point of view of treatment and prevention) of aid devices and orthoses? & XIV \\
\hline 15. & $\begin{array}{l}\text { When should TENS, acupuncture, balneotherapy and exercises in water, manual therapy } \\
\text { and patellar taping be used? }\end{array}$ & $\mathrm{XV}$ \\
\hline \multicolumn{3}{|c|}{ FOLLOW-UP } \\
\hline 16. & When is orthopedic treatment required in patients with $O A$ ? & XVI \\
\hline
\end{tabular}

OA, osteoarthritis; NSAIDs, non-steroidal anti-inflammatory drugs; SYSADOA, symptomatic slow acting drugs in OA. 
Inclusion and exclusion criteria

Clinical practice guidelines and consensus statements with recommendations for knee, hip and hand OA management endorsed by SIR, scientific societies belonging to the International League of Associations for Rheumatology (ILAR) (e.g. American College of Rheumatology - ACR, African League of Associations for Rheumatology - AFLAR, Asia-Pacific League Against Rheumatism - APLAR, Pan-American League of Associations for Rheumatology - PANLAR, European League Against Rheumatism - EULAR) or multidisciplinary task forces focused on OA management (Osteoarthritis Research Society International - OARSI and American Association of Orthopedic Surgeons AAOS), published in English or Italian between January $1^{\text {st }} 2005$ and April $30^{\text {th }} 2017$, were included.

Published studies which did not provide guidelines or consensus statements including randomized controlled trials (RCT)

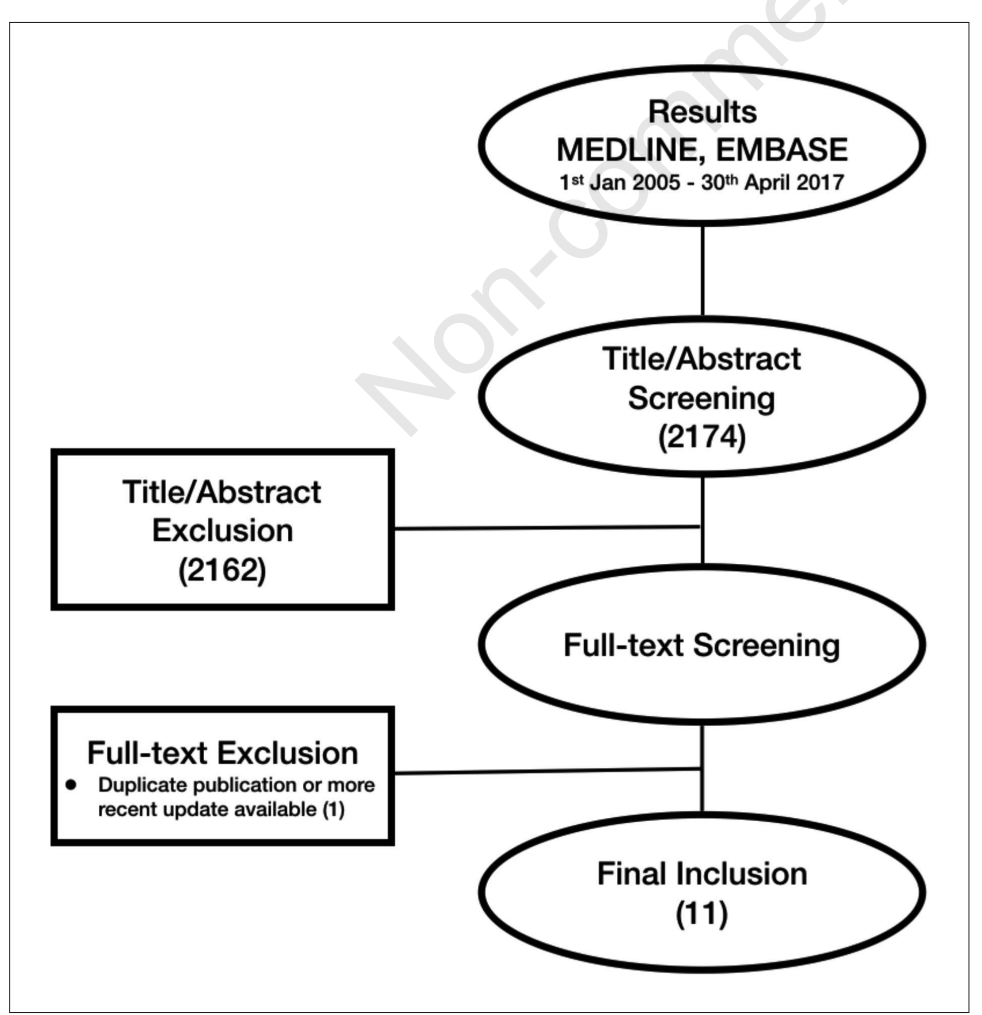

Figure 1 - Steps in the systematic review of guidelines on diagnosis and treatment of knee, hip and hand osteoarthritis. and uncontrolled trials, observational studies, editorials, commentaries, conference abstracts and narrative/systematic reviews were excluded. CPGs and consensus statements in languages other than English and Italian, which were non-original i.e. duplicated, adapted or updated from previous recommendations) and/or reported with poor methodology, and did not answer the key health questions, were excluded as well.

\section{Search strategy}

A systematic literature search according to the criteria detailed above was designed by the members of the Study Centre and performed by AA in Pubmed/Medline and Embase databases combining keywords for OA, drug names, and guidelines and consensus statements (Appendix). All search results were screened by 2 independent reviewers (AA, MM), and disagreements were resolved by consensus or by discussion involving the members of the working group. A flow chart of search results is presented in Figure 1.

\section{Appraisal of guideline quality}

Guideline quality was assessed by two raters (AA, MM), using the on-line Appraisal of Guidelines Research and Evaluation (AGREE) II instrument (10). The final AGREE score was not considered as a criteria for exclusion.

\section{Level of evidence and strength of recommendation}

Different grading systems for evidence were used across guidelines. In order to reconcile these differences, each guidelines' grading system was revised and the level of evidence of recommendation was rated according to the Oxford Levels of Evidence (http://www.cebm.net/oxfordcentre-evidence-based-medicine-levelsevidence-march-2009/) (Table II). In case of uncertainty, the rating of the recommendation based on the most updated evidence was considered.

\section{Evidence framework} and development of recommendations

The descriptive characteristics of the included guidelines (guideline developer, 
Table II - Guidance to categories of evidence and strength of guidelines based on the Oxford Levels of Evidence.

\begin{tabular}{|c|l|}
\hline Category & Evidence \\
\hline 1 & From meta-analysis of randomized controlled trials or from at least one randomized controlled trial \\
\hline 2 & From at least one controlled study without randomization or from at least one cohort study \\
\hline 3 & From at least one case-control study \\
\hline 4 & From case-series or poor-quality cohort and case-control studies \\
\hline 5 & From expert committee reports or opinions and/or clinical experience of respected authorities \\
\hline
\end{tabular}

topic, country, language, publication year, end-of-search date, grading systems) and AGREE scores were synthesized in tables (not shown).

For each key question, evidence tables containing guideline characteristics, recommendations, AGREE summary scores, and level of evidence and strength of recommendation according to the original grading system were prepared.

An Italian recommendation was developed by the endorsement or the adaptation and rewording of existing recommendations. This guidance was prepared in accordance with the AGREE reporting checklist (11). Additional methodological details may be found in the accompanying article (12).

\section{Peer review}

The draft of the guidelines developed by the working group was sent to external reviewers $(\mathrm{n}=18)$ for comment and rating. An online survey was performed via Google Forms between June $30^{\text {th }}$ and July $15^{\text {th }} 2018$ and feedback from respondents $(\mathrm{n}=12$, response rate $68 \%)$ was considered in order to finalize the guidelines. The final document underwent a peer review process through the journal.

\section{RESULTS}

\section{Key to understanding this guidance}

Each recommendation is presented with a level of evidence and strength of recommendation and is accompanied by a supporting text that is structured as follows:

Summary of guidelines: a synthesis of guidelines included in the original guidelines on OA identified from the systematic review.

Recommendation/supporting evidence: specific source guidelines that were used for adaptation.

Evidence for recommendation: the results of the panel's discussion of the adapted recommendation with regard to further specifications and comments on the sources used to develop the recommendation.

\section{Recommendations}

Eleven original clinical practice guidelines (CPGs) (7, 13-22) were selected and used to obtain the final set of 16 recommendations (Table III).

\section{RECOMMENDATION 1}

Findings indicative of OA

- Symptoms: painful and/or restricted movement, instability and regional

Table III - The final set of 16 guidelines for the management of knee, hip and hand OA with respective level of evidence.

\begin{tabular}{|c|l|c|}
\hline No. & Recommendation & \multicolumn{1}{c|}{$\begin{array}{l}\text { Level of } \\
\text { Evidence }\end{array}$} \\
\hline & $\begin{array}{l}\text { Findings indicative of OA: } \\
\text { Symptoms: painful and/or restricted movement, instability and regional pain. More persistent rest and night pain may } \\
\text { occur in advanced OA. OA symptoms are often episodic or variable in severity and slow to change. }\end{array}$ \\
1 & $\begin{array}{l}\text { Signs: crepitus, bony enlargement/deformity, absent or modest joint inflammation, bony ankylosis, only short-lived } \\
\text { morning stiffness, malalignment, and the presence of Heberden's nodes. } \\
\text { Clinical/family history: female sex, age over } 40 \text { years, menopausal status, family history, obesity, joint laxity, prior hand } \\
\text { injury, occupation or recreation-related usage. }\end{array}$ & $1-4$ \\
\hline
\end{tabular}




\begin{tabular}{|c|c|c|}
\hline No. & Recommendation & $\begin{array}{l}\text { Level of } \\
\text { Evidence }\end{array}$ \\
\hline 2 & $\begin{array}{l}\text { Imaging is not required to make the diagnosis in patients with typical presentation of OA. In atypical } \\
\text { presentations, imaging is recommended to help confirm the diagnosis of } \mathrm{OA} \text { and/or make alternative or } \\
\text { additional diagnoses. Imaging features do not predict non-surgical treatment response. }\end{array}$ & $2-4$ \\
\hline 3 & $\begin{array}{l}\text { If imaging is needed, conventional (plain) radiography should be used before other modalities because it is the current } \\
\text { gold standard for morphological assessment of OA. The recommended views are: A) [for the knee], weight bearing } \\
\text { and patello-femoral views, B) [for the hand] a postero-anterior radiograph of both hands on a single film/field of view. } \\
\text { Classical features are focal joint space narrowing, osteophyte, subchondral bone sclerosis and subchondral cysts. To } \\
\text { make additional diagnoses, soft tissues are best imaged by ultrasounds (US) or magnetic resonance imaging (MRI) } \\
\text { and bone by computed tomography (CT) or MRI. }\end{array}$ & $1-4$ \\
\hline 4 & $\begin{array}{l}\text { If a palpable effusion is present, synovial fluid should be aspirated and analysed to exclude inflammatory disease } \\
\text { and to identify urate and calcium pyrophosphate crystals. OA synovial fluid is typically non-inflammatory with } \\
<2000 \text { leukocytes/mm; if specifically sought, basic calcium phosphate crystals are often present. }\end{array}$ & 2 \\
\hline 5 & $\begin{array}{l}\text { Optimal management of } \mathrm{OA} \text { requires a combination of non-pharmacological and pharmacological treatment modalities } \\
\text { individualized to the patient's needs. }\end{array}$ & 5 \\
\hline 6 & $\begin{array}{l}\text { Treatment of hand, hip and knee OA should be individualized according to: 1) the wishes and expectations of the } \\
\text { individual, 2) localization, severity of structural change and type of OA, 3) risk factors (such as age, sex, obesity and } \\
\text { adverse mechanical factors), 4) presence of inflammation, 5) comorbidity and co-medication, 6) OA in other sites. }\end{array}$ & $1-4$ \\
\hline 7 & $\begin{array}{l}\text { Blood, urine or synovial fluid tests are not required for diagnosis of OA but may be required for differential } \\
\text { diagnosis. In OA patients with marked inflammatory symptoms and/or signs, especially involving atypical sites, } \\
\text { laboratory tests should be undertaken. }\end{array}$ & $1-2$ \\
\hline 8 & $\begin{array}{l}\text { Paracetamol (acetaminophen) (up to } 3 \mathrm{~g} / \text { day) is an effective initial oral analgesic for treatment of mild to } \\
\text { moderate pain. In elderly patients it should be preferred because of its relative safety in comparison with } \\
\text { NSAIDs. } \\
\text { The use of weak opioids in case of severe pain or no response, intolerance or contraindication to NSAIDs, } \\
\text { is recommended. Stronger opioids should only be used for the management of severe pain in exceptional } \\
\text { circumstances. } \\
\text { Duloxetine may be helpful for knee (and maybe hip) OA accompanied by chronic pain. }\end{array}$ & $1-4$ \\
\hline 9 & $\begin{array}{l}\text { Oral NSAIDs are recommended at the lowest effective dose and for the shortest duration in patients who } \\
\text { respond inadequately to paracetamol. NSAIDs (such as ibuprofen, diclofenac and naproxen) and selective COX- } \\
2 \text { inhibitors (including celecoxib and etoricoxib) are indicated in moderate pain. Higher doses of oral NSAIDs may } \\
\text { be indicated in more severe pain. } \\
\text { In patients with increased gastrointestinal risk, non-selective NSAIDs plus a proton-pump inhibitor, or a selective } \\
\text { COX-2 inhibitor, should be used. } \\
\text { In patients with increased cardiovascular risk, naproxen can be used; COX-2 inhibitors are contraindicated and } \\
\text { other non-selective NSAIDs should be used with caution. In nephropathic patients the use of NSAID and COX-2 } \\
\text { inhibitors should be avoided. }\end{array}$ & 1 \\
\hline 10 & $\begin{array}{l}\text { Topical pharmacological treatments are preferred over systemic treatments, especially for mild to moderate pain } \\
\text { and when only a few joints are affected. Topical NSAIDs and capsaicin gel are effective and safe treatments. } \\
\text { Patients with age }>75 \text { years should use topical rather than oral NSAIDs even though the analgesic response } \\
\text { decreases after } 1 \text { year of use. }\end{array}$ & $1-2$ \\
\hline 11 & $\begin{array}{l}\text { The accuracy of intra-articular injection depends on the joint and on the skills of the practitioner. Ultrasound- } \\
\text { guidance may improve accuracy and it is particularly recommended for joints that are difficult to access due to } \\
\text { the site itself, degree of deformity or obesity. } \\
\text { Hyaluronic Acid: intra-articular injection of hyaluronic acid of different molecular weights may give symptomatic } \\
\text { benefit with low toxicity and could help to reduce the NSAID use. } \\
\text { Steroids: intra-articular corticosteroid injection may be beneficial, providing fast pain relief in patients who suffer } \\
\text { painful relapses and who do not respond or have a contraindication to analgesics and NSAIDs. } \\
\text { Mesenchymal stem cells and/or platelet rich plasma: it is unclear if intra-articular injection of mesenchymal stem } \\
\text { cells or platelet-rich plasma can help to relieve pain associated with knee OA. }\end{array}$ & $1-5$ \\
\hline 12 & $\begin{array}{l}\text { In patients with symptomatic knee } O A \text {, glucosamine sulphate and chondroitin sulphate may have a beneficial } \\
\text { effect on symptoms. Structural effects, patients suitable for treatment and the cost to benefit ratio of the therapy } \\
\text { remain to be defined. }\end{array}$ & $1-2$ \\
\hline
\end{tabular}




\begin{tabular}{|c|c|c|}
\hline No. & Recommendation & $\begin{array}{l}\text { Level of } \\
\text { Evidence }\end{array}$ \\
\hline 13 & $\begin{array}{l}\text { Concerning patient education, lifestyle changes and therapeutic exercise: } \\
\text { Patients' education } \\
\text { Information, education and an individually tailored program, including long-term and short-term goals, intervention or } \\
\text { action plans to reduce the degenerative damage of the OA should be provided. People with hip and/or knee OA should } \\
\text { be taught a regular individualized (daily) exercise regimen and participate in self-management programs, strengthening, } \\
\text { low-impact aerobic exercises, and neuromuscular education. } \\
\text { Life styles } \\
\text { Patients with hip and knee OA, who are overweight, should be encouraged to lose weight and maintain their weight at a } \\
\text { lower level. People with hip or knee OA at risk of work disability should have access to vocational rehabilitation, including } \\
\text { counselling on modifiable work-related factors. } \\
\text { Exercise } \\
\text { The mode of delivery of exercise education should be selected according both to the preference of the person with hip or } \\
\text { knee OA and local availability. Patients with knee OA should participate in aerobic and/or resistance land-based and/or } \\
\text { aquatic exercise. }\end{array}$ & $1-3$ \\
\hline 14 & $\begin{array}{l}\text { Orthoses prevent the progression of degenerative changes and improve function. In hip and knee OA, the use of } \\
\text { assistive devices such as a walking-stick or crutches is suggested as a preventive measure. The use of appropriate and } \\
\text { comfortable shoes is recommended. } \\
\text { The combination of splints for thumb base } O A \text {, orthoses and exercise regimen reduce pain and improve functionality in } \\
\text { the short and long term and prevent/correct lateral angulation and flexion deformity. }\end{array}$ & $1-2$ \\
\hline 15 & $\begin{array}{l}\text { Concerning TENS, acupuncture, balneotherapy and exercises in water, manual therapy and patellar taping: } \\
\text { TENS: transcutaneous electrical nerve stimulation (TENS) may help with short-term pain control in some patients with hip } \\
\text { or knee OA. } \\
\text { Acupuncture: the usefulness in patients with symptomatic OA of the knee and hip remains to be defined. } \\
\text { Balneotherapy and exercises in water are effective for relieving symptoms in hip and knee (and hand) OA. } \\
\text { Manual therapy/Taping: it is unclear if manual therapy can be useful in patients with symptomatic osteoarthritis of the } \\
\text { knee. The use of bandage tape may help to reduce pain in patients with joint instability knee OA. }\end{array}$ & $1-4$ \\
\hline 16 & $\begin{array}{l}\text { Orthopedic surgery should be considered in patients with radiographic evidence of } \mathrm{OA} \text {, who have marked disability, } \\
\text { reduced quality of life and pain refractory to other treatments. }\end{array}$ & 5 \\
\hline
\end{tabular}

pain. More persistent rest and night pain may occur in advanced OA. OA symptoms are often episodic or variable in severity and slow to change.

- Signs: crepitus, bony enlargement/deformity, absent or modest joint inflammation, bony ankylosis, only shortlived morning stiffness, malalignment, and the presence of Heberden's nodes.

- Clinical/family history: female sex, age over 40 years, menopausal status, family history, obesity, joint laxity, prior hand injury, occupation or recreationrelated usage.

(Level of Evidence: 1-4)

Summary of guidelines. Most symptoms and signs are in common with all forms of OA. However, some of them are characterized by exclusive (e.g. nodules of Heberden in hand OA) or highly frequent (varus/valgus in the OA of the knees or lateral deviation of interphalangeal joints, subluxation and adduction of thumb base) changes (level 1-3). Heberden nodules are a risk factor for the occurrence of $\mathrm{OA}$ in other sites, especially in the knee (level 1-2) and hip (level 2).

Recommendation/supporting evidence. EULAR 2010, EULAR 2009.

Evidence for recommendation. The panel suggested slight changes to the original statements. Joint inflammation was substituted by effusion and tenderness in order to highlight the clinical difference from inflammatory arthritis. However, the panel underlines that major signs of joint inflammation may infrequently occur in $\mathrm{OA}$ in typical sites. Higher bone density and greater forearm muscle strength were deleted: even if they are associated with increased risk of osteoarthritis (23), the relationship between these conditions most likely takes into account potential covariates (24).

External reviewers median score 10/10 ( $100 \%$ of scores $\geq 7$ ) 


\section{RECOMMENDATION 2}

Imaging is not required to make the diagnosis in patients with typical presentation of OA. In atypical presentations, imaging is recommended to help confirm the diagnosis of OA and/or make alternative or additional diagnoses. Imaging features do not predict non-surgical treatment response. (Level of Evidence: 2-4)

Summary of guidelines. According to EULAR recommendation, subjects with clinical manifestations typical of OA (in any district) do not need special imaging investigations (level 3). Imaging is useful only in those patients whose clinical features are not entirely consistent with OA or where an overlap with other diseases is suspected (level 4). Radiological features do not provide information on the response to nonsurgical therapy (level 2-3) and therefore $\mathrm{X}$-rays should not be performed for this purpose.

Recommendation/supporting evidence. EULAR 2017.

Evidence for recommendation. The panel agreed upon imaging (X-rays, computed tomography, ultrasounds and magnetic resonance) as having a role in typical and atypical OA as indicated by EULAR guidelines. However, X-rays have a pivotal role in OA staging, especially in knee and hip (25). The external review panel is less prone to adhere to this general point of view since many specialists deem imaging an added value in the management of patients with OA in clinical practice.

External reviewers median score $8 / 10$ $(67 \%$ of scores $\geq 7)$.

\section{RECOMMENDATION 3}

If imaging is needed, conventional (plain) radiography should be used before other modalities because it is the current gold standard for morphological assessment of OA. The recommended views are: A) [for the knee], weight bearing and patellofemoral views, B) [for the hand] a postero-anterior radiograph of both hands on a single film/field of view. Classical features are focal joint space narrowing, osteophyte, subchondral bone sclerosis and subchondral cysts. To make additional diagnoses, soft tissues are best imaged by Ultrasounds (US) or Magnetic Resonance Imaging (MRI) and bone by Computed Tomography (CT) or MRI.

(Level of Evidence: 1-4)

Summary of guidelines. When required, the first radiological examination to be carried out regardless of the anatomical district involved is the radiography (level 1-2). Elementary lesions are in common to all OA forms; in hand OA, subchondral cysts must also be sought. In addition, radiographs must be obtained in conventional projections and always performed bilaterally (level 1-3). Only if the bone or periarticular tissues are to be further investigated, is it possible to take into consideration the other above-mentioned imaging modalities (level 3-4).

Recommendation/supporting evidence. EULAR 2009, EULAR 2010, EULAR 2017.

Evidence for recommendation. The panel agreed upon the plain radiography views to be considered in knee and hand OA. The standard hip X-ray examination includes an anteroposterior and a lateral image. Alternative views could be performed in order to better investigate the knee patellofemoral OA (26) or the early phase (27). External reviewers median score 10/10 ( $100 \%$ of scores $\geq 7$ ).

\section{RECOMMENDATION 4}

If a palpable effusion is present, synovial fluid should be aspirated and analysed to exclude inflammatory disease and to identify urate and calcium pyrophosphate crystals. OA synovial fluid is typically non-inflammatory with $<2000$ leukocytes $/ \mathrm{mm}^{3}$; if specifically sought, basic calcium phosphate crystals are often present.

(Level of Evidence: 2)

Summary of guidelines. This recommendation was accepted without any change from the original one. 
Recommendation/supporting evidence. EULAR 2010.

Evidence for recommendation. The panel agreed that synovial fluid analysis should be always performed in new onset swollen joints or if the effusion is not clearly related to OA.

External reviewers median score $9 / 10$ ( $75 \%$ of scores $\geq 7$ ).

\section{RECOMMENDATION 5}

Blood, urine or synovial fluid tests are not required for diagnosis of OA but may be required for differential diagnosis. In OA patients with marked inflammatory symptoms and/or signs, especially involving atypical sites, laboratory tests should be undertaken.

(Level of Evidence: 1-2)

Summary of guidelines. In general, laboratory tests (blood count, inflammation, urinalysis or synovial fluid) are used for differential diagnostic purposes. In particular they are useful for excluding chronic or crystal-induced arthropathies.

Recommendation/supporting evidence. EULAR 2009, EULAR 2010.

Evidence for recommendation. The panel agreed upon the laboratory test role in OA. The indications for the examination of the synovial fluid were previously discussed (see recommendation 6).

External reviewers median score 10/10 $(100 \%$ of scores $\geq 7)$.

\section{RECOMMENDATION 6}

Optimal management of $\mathrm{OA}$ requires a combination of non-pharmacological and pharmacological treatment modalities individualized to the patient's needs.

(Level of evidence: 5)

Summary of guidelines. The search identified 3 CPGs that addressed the optimal management of OA. According to EULAR, SIR and OARSI, clinicians must combine pharmacological and non-pharmacological treatment modalities in $\mathrm{OA}$ management.
In particular, SIR and EULAR emphasize the opportunity to adapt this management to the needs of the individual patient affected by hand OA.

Recommendation/supporting evidence. SIR 2013, OARSI 2008, EULAR 2007.

Evidence for recommendation. The panel agreed upon the role of the pharmacological and non-pharmacological treatment that should be individualized.

External reviewers median score 10/10 ( $100 \%$ of scores $\geq 7$ ).

\section{RECOMMENDATION 7}

Treatment of hand, hip and knee OA should be individualized according to:

1) the wishes and expectations of the individual;

2) localization, severity of structural change and type of $\mathrm{OA}$;

3) risk factors (such as age, sex, obesity and adverse mechanical factors);

4) presence of inflammation;

5) comorbidity and co-medication;

6) OA in other sites.

(Level of Evidence: 1-4)

Summary of guidelines. The search identified 3 CPGs addressing individualized treatment of OA.

The above factors must be taken into consideration especially in the presence of knee and hip OA (level 1). It seems reasonable to extend the concept to hand OA (level 3,4). The importance of customizing the treatment according to the type of structural lesion is particularly relevant in hand OA. Clinicians should establish which type of hand OA (nodal, erosive or post-traumatic) they are facing (level 3, 4).

Recommendation/supporting evidence. EULAR 2013, SIR 2013, EULAR 2007.

Evidence for recommendation. The panel agreed upon the issues to consider in tailoring OA treatment. Minor changes with respect to the original CPGs were made in order make the list clearer without any redundancy.

External reviewers median score 10/10 ( $100 \%$ of scores $\geq 7$ ). 


\section{RECOMMENDATION 8}

Paracetamol (acetaminophen) (up to $3 \mathrm{~g} /$ day) is an effective initial oral analgesic for treatment of mild to moderate pain. In elderly patients it should be preferred because of its relative safety in comparison with NSAIDs.

The use of weak opioids in case of severe pain or no response, intolerance or contraindication to NSAIDs, is recommended. Stronger opioids should only be used for the management of severe pain in exceptional circumstances.

Duloxetine may be helpful for knee (and maybe hip) OA accompanied by chronic pain.

(Level of Evidence: 1-4)

Summary of guidelines. The use of paracetamol remains controversial. In most guidelines taken into consideration, paracetamol should be the first analgesic to use (level 1a-1b), but the AAOS meta-analysis was inconclusive about its actual usefulness (level 4).

All scientific societies recommend the use of weak opioids (in particular tramadol) whenever paracetamol is not very effective and/or NSAIDs are contraindicated (due to cardiovascular or gastroenterological risk and lack of efficacy) (level 2).

Duloxetine should be considered only in subjects with knee OA (level 2).

Recommendation/supporting evidence. PANLAR 2016, OARSI 2014, SIR 2013, ACR 2012, OARSI 2008, EULAR 2007.

Evidence for recommendation. The panel agreed upon the use of paracetamol as first line analgesic. According to the Italian acetaminophen summary of product characteristics, the maximum daily dose of acetaminophen is $3 \mathrm{~g} /$ day.

According to the panel, it is reasonable to suppose that duloxetine may have a similar effect on hip OA (28). Furthermore, other centrally acting analgesic agents will soon be included among the analgesics for knee and hip OA (29).

Stronger opioids are not recommended in hand $\mathrm{OA}$.

The panel ascertain that this recommendation is substantially overlapping with the therapeutic algorithm recommended by the ESCEO (30).

External reviewers median score $8 / 10$ ( $92 \%$ of scores $\geq 7$ ).

\section{RECOMMENDATION 9}

Oral NSAIDs are recommended at the lowest effective dose and for the shortest duration in patients who respond inadequately to paracetamol. NSAIDs (such as ibuprofen, diclofenac and naproxen) and selective COX-2 inhibitors (including celecoxib and etoricoxib) are indicated in moderate pain. Higher doses of oral NSAIDs may be indicated in more severe pain.

In patients with increased gastrointestinal risk, non-selective NSAIDs plus a protonpump inhibitor, or a selective COX-2 inhibitor, should be used.

In patients with increased cardiovascular risk, naproxen can be used; COX-2 inhibitors are contraindicated and the other non-selective NSAIDs should be used with caution.

In nephropathic patients the use of NSAID and COX-2 inhibitors should be avoided. (Level of Evidence: 1)

Summary of guidelines. The use of NSAIDs is recommended in all forms of OA. Patient evaluation must be performed beforehand to assess cardio-vascular, gastro-intestinal and renal risks. NSAIDs have a high level of evidence (level 1) in those subjects who have failed the therapy with paracetamol. Recommendation/supporting evidence. PANLAR 2016, AAOS 2013, SIR 2013, ACR 2012, OARSI 2008, EULAR 2007. Evidence for recommendation. The panel agreed in limiting NSAID use to the lowest effective dose. When selecting NSAIDs, it must be taken into account that the European Medicines Agency's (EMA) Pharmacovigilance Risk Assessment Committee (PRAC) found that the effects of systemic diclofenac on the heart and circulation are similar to those of selective COX-2 inhibitors (see EMA Safety Advice 592685/2013). Clinicians must be careful when co-administrating acetylsalicylic acid (ASA) and ibuprofen in patients with a high cardiovascular risk. Ibuprofen antagonizes the anti-platelet 
action of ASA (low dose) with consequent reduction of cardio-protective effect. This interaction is minimal if ibuprofen is taken (occasionally) 2 hours after the administration of ASA. Therefore, ASA should be administered 2 hours before the first daily dose of ibuprofen, especially in therapeutic regimens involving triple daily administration (31). In nephropathic patients, the use of NSAIDs and COX-2 should be avoided (32). Naproxen has a safer cardiovascular profile than other NSAIDs. However, this benefit is limited in high-risk cardiovascular patients $(33,34)$.

External reviewers median score 10/10 (92\% of scores $\geq 7$ ).

\section{RECOMMENDATION 10}

Topical pharmacological treatments are preferred over systemic treatments, especially for mild to moderate pain and when only a few joints are affected. Topical NSAIDs and capsaicin gel are effective and safe treatments.

Patients with age $>75$ years should use topical rather than oral NSAIDs, even though the analgesic response decreases after 1 year of use.

(Level of Evidence: 1-2)

Summary of guidelines. Topical capsaicin is effective in both knee and hand OA (level 2 and 1 respectively); topical NSAIDs are equally effective (level 1 ) even if over time their action can wane (level 1).

Recommendation/supporting evidence. PANLAR 2016, SIR 2013, ACR 2012, OARSI 2008, EULAR 2007.

Evidence for recommendation. The panel expects, in the next years, the identification of predictors responding well to topical treatment (35).

External reviewers median score $8 / 10$ ( $100 \%$ of scores $\geq 7)$.

\section{RECOMMENDATION 11}

The accuracy of intra-articular injection depends on the joint and on the skills of the practitioner. Ultrasound-guidance may improve accuracy and it is particularly rec- ommended for joints that are difficult to access due to the site itself, degree of deformity or obesity.

- Hyaluronic Acid: intra-articular injection of hyaluronic acid of different molecular weights may give symptomatic benefit with low toxicity and could help to reduce NSAID use.

- Steroids: intra-articular corticosteroid injection may be beneficial, providing fast pain relief in patients who suffer painful relapses and who do not respond or have a contraindication to analgesics and NSAIDs.

- Mesenchymal stem cells and/or platelet rich plasma: it is unclear if intra-articular injection of mesenchymal stem cells or platelet-rich plasma can help to relieve pain associated with knee OA.

(Level of Evidence: 1-5)

Summary of guidelines. Imaging examination (in particular ultrasonography), as well as the experience of the performer, increases the success rate of the infiltrative procedure (level 3-5).

The effect of hyaluronic acid (HA) has been verified in subjects with knee, hand and hip OA. Although a certain tendency towards a beneficial effect of this therapy (in particular in knee OA) and a possible effect on delay of the eventual prosthesis have been detected, the studies conducted are burdened by small effect sizes (level 1-2).

Steroid infiltration has a relevant but transient analgesic effect in all forms of OA (level 2). The use of a long-acting steroid is useful in reducing painful exacerbations, particularly in the trapezium-metacarpal joint (level 2).

Infiltrative therapy with platelet gel and mesenchymal stem cells is to be considered only in case of symptomatic knee OA (level 2-3), given the need to confirm its effectiveness with good quality studies.

Recommendation/supporting evidence. EULAR 2017, PANLAR 2016, SIR 2013, AAOS 2013, EULAR 2007, OARSI 2008. Evidence for recommendation. The panel agreed it would be worthwhile to consider HA injections in selected knee and hip OA. 
Nevertheless, the patients who may benefit from the treatment are not accurately defined. Moreover, clinically relevant structure modification and cost-benefit ratio have not been established. Studies and meta-analyses are ongoing to examine this theme more deeply. A panel of Italian experts agreed that HA injections are useful for mild to moderate hip and knee OA (36). The panel underline the possible different roles in pain relief of intra-articular steroids and HA. The former has a rapid effect, especially when signs of inflammation are detectable, while the latter is supposed to induce a long-standing pain relief.

External reviewers median score 9/10 ( $83 \%$ of scores $\geq 7$ ).

\section{RECOMMENDATION 12}

In patients with symptomatic knee OA, glucosamine sulphate and chondroitin sulphate may have beneficial effect on symptoms. Structural effects, patients suitable for treatment and the cost to benefit ratio of the therapy remain to be defined.

(Level of Evidence: 1-2)

Summary of guidelines. Oral treatment with glucosamine and/or chondroitin sulphate in knee OA can be taken into consideration even if the guidelines of the various scientific societies are discordant (level 1-2). The small-pooled effect sizes contribute to this heterogeneity of judgment, as well as inconsistency in the results between the industry, sponsorship and independent trials, and the heterogeneity among studies. It is hypothesized that the patients who could benefit most are the younger ones (level 2) or those with more severe symptoms (level 1). The expected effects include pain reduction and/ or joint function improvement (level 1). Symptomatic Slow Acting Drugs in OA. (SYSADOA) may be suspended if no benefit is observed within six months (level 1). In hip or hand OA, the results of the efficacy studies show even more heterogeneity, making the guidelines ambiguous (level 1). Recommendation/supporting evidence. PANLAR 2016, OARSI 2014, AAOS 2013, ACR 2012, OARSI 2008.
Evidence for recommendation. The panel agreed upon the possible symptomatic effect of glucosamine and/or chondroitin sulphate in symptomatic knee OA. However, it considers the opinion of the OARSI 2014 panel on this issue as the most balanced. Moreover, both glucosamine and chondroitin sulphate have a pain relief effect in symptomatic knee OA, but knowledge about their structural effects and cost-benefit ratio is insufficient. As the dosage and frequency of administration are not standardized, the panel suggest taking into account the information included in the leaflet.

External reviewers median score 9/10 ( $92 \%$ of scores $\geq 7$ ).

\section{RECOMMENDATION 13}

Concerning patient education, lifestyle changes and therapeutic exercise:

\section{Patient's education}

Information, education and an individually tailored program, including long-term and short-term goals, intervention or action plans to reduce the degenerative damage of the OA should be provided. People with hip and/or knee OA should be taught a regular individualized (daily) exercise regimen and participate in self-management programs, strengthening, low-impact aerobic exercises, and neuromuscular education.

\section{Life styles}

Patients with hip and knee OA, who are overweight, should be encouraged to lose weight and maintain their weight at a lower level. People with hip or knee OA at risk of work disability should have access to vocational rehabilitation, including counselling about modifiable work-related factors.

\section{Exercise}

The mode of delivery of exercise education should be selected according both to the preference of the person with hip or knee OA and local availability. Patients with knee OA should participate in aerobic and/or resistance land-based and/or aquatic exercise. (Level of Evidence: 1-3)

Summary of guidelines. Indications regarding patient education refer mainly to sub- 
jects with knee or hip OA (level 1). The education program includes management of the exercises using appropriate walking aids where necessary (e.g. walking-sticks, crutches and shoe adjustments) and other measures to prevent the progression of joint damage (level 1).

Lifestyle changes consist not only in a balanced diet but also in a modulation of domestic and work activities (level 1-3).

The indications regarding the therapeutic exercises concern exclusively the knee and hip OA (level 1a). Before establishing the exercise plan, it is essential to evaluate the ability to perform activities of daily living (ADLs), instruct in joint protection techniques and provide assistive devices, as needed (level $1)$. Weight loss through aerobic activity in water or not is to be set for overweight or obese subjects (level 1). The programs must be flexible and easily modifiable according to the patient's needs and to the objectives that he/she actually achieves over time (level 1).

Recommendation/supporting evidence. PANLAR 2016, AAOS 2013, EULAR 2013, OARSI 2008, ACR 2012.

Evidence for recommendation. The panel agreed upon the importance of patients' education, exercise and appropriate lifestyle. In particular, changes in work-related factors encompass altering work behavior, modifying tasks or work hours, use of assistive technology, improvement of workplace and support from management, colleagues and family towards employment.

External reviewers median score 10/10 $(100 \%$ of scores $\geq 7)$.

\section{RECOMMENDATION 14}

Orthoses prevent the progression of degenerative changes and improve function. In hip and knee OA, the use of assistive devices such as a walking-stick or crutches is suggested as a preventive measure. The use of appropriate and comfortable shoes is recommended.

The combination of splints for thumb base $\mathrm{OA}$, orthoses and exercise regimen reduce pain and improve functionality in the short and long term and prevent/correct lateral angulation and flexion deformity.

(Level of Evidence: 1-2)
Summary of guidelines. Over the years the level of evidence for these guidelines has increased from the first pronouncements of OARSI. This statement refers to the non-pharmacological and pre-surgical treatment of OA. In addition, the indications given are to be considered valid only for the aids and orthoses listed. The highest degree of evidence is recorded for the use of shoes in subjects with knee and hip $\mathrm{OA}$ and for the orthosis for hand OA (i.e. rhizarthrosis) (level 1).

Recommendation/supporting evidence. PANLAR 2016, AAOS 2013, SIR 2013, EULAR 2013, OARSI 2008, EULAR 2007.

Evidence for recommendation. The panel underline the importance of proper walking-stick use (e.g. in the contralateral hand and with the height adjusted to the level of the greater trochanter, and the elbow bent at an angle of 25 to 30 degrees).

External reviewers median score 9/10 ( $92 \%$ of scores $\geq 7$ ).

\section{RECOMMENDATION 15}

About TENS, acupuncture, balneotherapy and exercises in water, manual therapy and patellar taping:

- TENS: Transcutaneous electrical nerve stimulation (TENS) may help with short-term pain control in some patients with hip or knee OA.

- Acupuncture: the usefulness in patients with symptomatic OA of the knee and hip remains to be defined.

- Balneotherapy and exercises in water are effective for relieving symptoms in hip and knee (and hand) OA.

- Manual therapy/Taping: It is unclear if manual therapy can be useful in patients with symptomatic osteoarthritis of the knee. The use of bandage tape may help to reduce pain in patients with joint instability knee OA.

(Level of Evidence: 1-4)

Summary of guidelines. TENS and taping indications are extensible to all forms of OA under examination in this review (level 1a and 1b). Acupuncture and manual 
therapy have been investigated with many studies but the ACR and the AAOS positions about their inconclusive effect prevail (level 4).

The effectiveness of balneotherapy and exercises in water was investigated in knee (level 1) and hip (level 1 only for exercises in water) $\mathrm{OA}$.

Recommendation/supporting evidence. PANLAR 2016, AAOS 2013, SIR 2013, ACR 2012, OARSI 2008.

Evidence for recommendation. The panel emphasize that both balneotherapy and exercises in water are complementary therapies. Balneotherapy is defined as bathing in natural mineral or thermal water without exercise (37). It encompasses the use of mineral waters (gas cans and peloids). Aquatic exercise (or hydrotherapy) is a therapeutic modality that is performed under the guidance of a qualified physiotherapist. (38). The possible efficacy of balneotherapy in hand OA is supported by recent systematic review (39).

External reviewers median score 9/10 ( $92 \%$ of scores $\geq 7$ ).

\section{RECOMMENDATION 16}

Orthopedic surgery should be considered in patients with radiographic evidence of OA, who have marked disability, reduced quality of life and pain refractory to other treatments.

(Level of Evidence: 5)

Summary of guidelines. The presence of one or more of the listed factors makes a surgical solution necessary. The surgical solution depends on the affected joint and on additional accessory lesions (e.g. tendons, ligaments) (level 5).

Recommendation/supporting evidence. PANLAR 2016, SIR 2013, AAOS 2013, OARSI 2008, EULAR 2007.

Evidence for recommendation. The panel is aware that, at the moment, there is no general agreement about the conditions that should lead to the orthopedic surgery.

External reviewers median score 10/10 ( $92 \%$ of scores $\geq 7$ ).

\section{DISCUSSION}

Even though in the past the SIR provided some CPGs on the management of OA in different sites, this is the first set of adapted guidelines endorsed by SIR that deals with knee, hip and hand OA taken together. The CPGs included in the process of adaptation were developed by international societies of rheumatology belonging to ILAR (ACR, AFLAR, APLAR, PANLAR, EULAR) or with the main commitment to manage OA (OARSI and AAOS). Merging guidelines concerning different joints (in terms of size and mechanical load that they have to bear) make the adapted guidelines inconsistent. It should be noted that this approach, which may seem disadvantageous given the possible different etiology of hand vs. knee and hip OA, has become common in CPGs published in recent years $(13,14,16$, $18)$. We agree with this approach, which makes it possible to have a holistic view of OA management.

$\mathrm{OA}$ is one of the most common chronic diseases and, as a consequence, the emission of OA-related CPGs is continuous. The main limitation of the process of adaptation is related to the availability of recent CPGs on the topic: the source CPGs included in our adapted guidelines were published in a time frame of 10 years. After April 30, 2017 (the end date of our systematic literature search for CPGs included in the ADAPTE process), Kloppenburg et al. published the updated EULAR recommendation for hand OA management (40). There is a substantial agreement between the new EULAR guidelines and our adapted guidelines about the overarching principles and the recommendations. However, some differences are present. The main difference concerns the use of paracetamol as a first line analgesic. The latest EULAR CPGs suggest using paracetamol in selected patients (when NSAIDs are contraindicated) because of its limited efficacy, emerging from recent clinical trials, together with some safety concerns. Moreover, the EULAR CPGs highlight the usefulness of oral chondroitin sulphate for pain relieving and function improvement (level of evidence 
1). The conditions leading to surgery could be clinical (pain not relieved by conservative treatment) or structural (presence of abnormalities restricting the function) (level of evidence 5).

In order to standardize knee, hip and hand OA management in Italy, SIR will disseminate these guidelines through national congresses, courses and educational sessions for health care providers. The implementation and dissemination of these CPGs is expected to improve treatment and prognosis of knee, hip and hand OA.

\section{Plans for updating}

These guidelines were endorsed by SIR as guides only and they do not substitute the individual clinicians' judgment, since they may not apply to all patients and all clinical situations. The SIR plans to review and update these guidelines in the future to determine if they remain current and to take heed of future treatments or advances in the management of knee, hip and hand OA.
Acknowledgements: the authors acknowledge all the external reviewers (in alphabetical order of those who gave consent for publication): Arioli G, Berruto M, Bianchi G, De Lucia O, Checchia GA, Mannoni A, Mascheroni G, Qarri D, Ramonda R, Sakellariu G, Tonolo S, Wolenski L. We are grateful to Solazzo T. as part of SIR secretariat for administrative support.

Contributions: all authors were involved in drafting the article or revising it critically for important intellectual content, and all authors approved the final version for publication. Conception and design: CS, NU, IP, MM, AB, SP, AA. Systematic review search and CPGs rating: AA, MM. Analysis and discussion of the guidelines: CS, NU, IP, MM, AB, SP, AA, AF, FI, FS.

Conflict of interests: AA, NU, SP declare a grant from SIR. All other authors have declared no conflict of interest.

\section{APPENDIX}

\section{Search Strategy}

Results were filtered for Published in the last 10 years (Jan 2017-Jul 2017).

Date of Searching Jul 2017.

OSTEOARTHRITIS

DATABASE: Medline via Pubmed (1)

"Osteoarthritis"[Mesh]

AND

("Practice Guideline" [Publication Type] OR "Practice Guidelines As Topic" [MeSH Terms] OR ((“Practice (Birm)" [Journal] OR "practice" [All Fields]) AND "guidelines as topic" [MeSH Terms]) OR Practice Guideline [Text Word] OR Practice Guidelines [Text Word] OR "Guideline" [Publication Type] OR "Guidelines As Topic" [MeSH Terms] OR "guidelines as topic" [MeSH Terms] OR Guideline [Text Word] OR Guidelines [Text Word] OR "Consensus Development Conference" [Publication Type] OR ((“consensus development conference" [Publication Type] OR "consensus development conferences as topic" [MeSH Terms] OR "consensus development conference" [All Fields])

AND

Topic[All Fields]) OR "consensus" [MeSH Terms] OR Consensus [Text Word] OR Recommendation [Text Word] OR Recommendations [Text Word] OR "practice guidelines as topic" [MeSH Terms] OR
Best Practice [Text Word] OR "practice guidelines as topic" [MeSH Terms] OR Best Practices [Text Word]) AND

(“2007/01/01”[PDat] : “2017/07/12”[PDat])

\section{Results 1273}

DATABASE: Medline via Pubmed (2)

("Osteoarthritis" [Mesh] OR "Osteoarthritis" [All Fields])

AND

("Practice Guideline" [Publication Type] OR "Practice Guidelines As Topic" [MeSH Terms] OR (("Practice (Birm)" [Journal] OR "practice"[All Fields]) AND "guidelines as topic" [MeSH Terms]) OR Practice Guideline [Text Word] OR Practice Guidelines [Text Word] OR "Guideline" [Publication Type] OR "Guidelines As Topic" [MeSH Terms] OR "guidelines as topic" [MeSH Terms] OR Guideline [Text Word] OR Guidelines [Text Word] OR "Consensus Development Conference" [Publication Type] OR 
((“consensus development conference" [Publication Type] OR "consensus development conferences as topic" [MeSH Terms] OR "consensus development conference" [All Fields])

AND

Topic [All Fields]) OR "consensus" [MeSH Terms] OR Consensus [Text Word] OR Recommendation [Text Word] OR Recommendations [Text Word] OR "practice guidelines as topic" [MeSH Terms] OR Best Practice [Text Word] OR "practice guidelines as topic" [MeSH Terms] OR Best Practices [Text Word]) AND

(“2007/01/01” [PDat] : "2017/07/12” [PDat])

\section{Results 2022}

\section{DATABASE: Embase}

\#1 AND (2007:py OR 2008:py OR 2009:py OR 2010:py OR 2011:py OR 2012:py OR 2013:py OR

\section{REFERENCES}

1. Cross M, Smith E, Hoy D, et al. The global burden of hip and knee osteoarthritis: estimates from the global burden of disease 2010 study. Ann Rheum Dis. 2014; 73: 1323-30.

2. Muraki S, Tanaka S, Yoshimura N. Epidemiology of knee osteoarthritis. OA Sports Medicine 2013; $1: 3$.

3. Kaur J. A comprehensive review on metabolic syndrome. Cardiol Res Pract. 2014; 2014: 943162-21.

4. Litwic A, Edwards MH, Dennison EM, Cooper C. Epidemiology and burden of osteoarthritis. Br Med Bull. Oxford University Press 2013; 105: 185-99.

5. Punzi L, Canesi B, Carrabba M, et al. [Italian consensus on Eular 2003 recommendations for the treatment of knee osteoarthritis]. Reumatismo. 2004; 56: 190-201.

6. Punzi L, Doherty M, Zhang W, Cimmino MA, Carrabba M, Frizziero L, et al. [Italian consensus on EULAR recommendations 2005 for the management of hip osteoarthritis]. Reumatismo. 2006; 58: 301-9.

7. Manara M, Bortoluzzi A, Favero M, et al. Italian Society for Rheumatology recommendations for the management of hand osteoarthritis. Reumatismo. 2013; 65:167-85.

8. Fervers B, Burgers JS, Haugh MC, et al. Adaptation of clinical guidelines: literature review and proposition for a framework and procedure. Int J Qual Health Care. 2006; 18: 167-76.

9. Fervers B, Burgers JS, Voellinger R, et al. Guideline adaptation: an approach to enhance efficiency in guideline development and im- 2014:py OR 2015:py OR 2016:py OR 2017:py) AND 'osteoarthritis'/de AND 'human'/de AND ('conference paper'/it OR 'conference review'/it OR 'review'/it) AND ('adverse drug reaction'/lnk OR 'complication'/lnk OR 'diagnosis'/lnk OR 'disease management'/lnk OR 'drug analysis'/lnk OR 'drug combination'/lnk OR 'drug comparison'/lnk OR 'drug dose'/lnk OR 'drug interaction'/lnk OR 'drug therapy'/lnk OR 'intraarticular drug administration'/lnk OR 'intravenous drug administration'/ lnk OR 'oral drug administration'/lnk OR 'pharmacology'/lnk OR 'prevention'/lnk OR 'side effect'/ lnk OR 'therapy'/lnk OR 'topical drug administration'/lnk) AND ('evidence based practice'/de OR 'practice guideline'/de OR 'systematic review'/de OR 'systematic review (topic)'/de) AND [embase]/ lim NOT [medline]/lim

\section{Results 155}

prove utilisation. BMJ Qual Saf. 2011; 20: 228-36.

10. Brouwers MC, Kho ME, Browman GP, et al. AGREE II: advancing guideline development, reporting and evaluation in health care. CMAJ. 2010; 182: E839-42.

11. Brouwers MC, Kerkvliet K, Spithoff K, AGREE Next Steps Consortium. The AGREE Reporting Checklist: a tool to improve reporting of clinical practice guidelines. BMJ. 2016; 352: i1152.

12. Manara M, Ughi N, Ariani A, et al. Providing updated guidelines for Italian rheumatologists with the ADAPTE methodology: a project of the Italian Society for Rheumatology. [In press].

13. Sakellariou G, Conaghan PG, Zhang W, et al. EULAR recommendations for the use of imaging in the clinical management of peripheral joint osteoarthritis. Ann Rheum Dis. BMJ. 2017 [epub ahead of print].

14. Rillo O, Riera H, Acosta C, et al. PANLAR Consensus Recommendations for the Management in Osteoarthritis of Hand, Hip, and Knee. J Clin Rheumatol. 2016; 22: 345-54.

15. McAlindon TE, Bannuru RR, Sullivan MC, et al. OARSI guidelines for the non-surgical management of knee osteoarthritis. Osteoarthr Cartilage OARS Osteoarthr Res Soc. 2014; 363-88.

16. Fernandes L, Hagen KB, Bijlsma JWJ, et al. EULAR recommendations for the non-pharmacological core management of hip and knee osteoarthritis. BMJ. 2013; 1125-35.

17. Brown GA. AAOS clinical practice guideline: Treatment of osteoarthritis of the knee: Evidence-based guideline. J Am Acad Orthop Surg. 2013; 21: 577-9. 
18. Hochberg MC, Altman RD, April KT, et al. American College of Rheumatology 2012 recommendations for the use of nonpharmacologic and pharmacologic therapies in osteoarthritis of the hand, hip, and knee. Arthritis Care Res (Hoboken). 2012; 64: 465-74.

19. Zhang W, Moskowitz RW, Nuki G, Abramson S, Altman RD, Arden NK, et al. OARSI recommendations for the management of hip and knee osteoarthritis, Part II: OARSI evidencebased, expert consensus guidelines. Osteoarthr Cartil. 2008; 16: 137-62.

20. Zhang W, Doherty M, Leeb BF, et al. EULAR evidence based recommendations for the management of hand osteoarthritis: report of a Task Force of the EULAR Standing Committee for International Clinical Studies Including Therapeutics (ESCISIT). Ann Rheum Dis. 2007; 66: 377-88.

21. Zhang W, Doherty M, Leeb BF, et al. EULAR evidence-based recommendations for the diagnosis of hand osteoarthritis: report of a task force of ESCISIT. Ann Rheum Dis. 2009; 68: 8-17.

22. Zhang W, Doherty M, Peat G, et al. EULAR evidence-based recommendations for the diagnosis of knee osteoarthritis. Ann Rheum Dis. 2010; 69: 483-9.

23. Nevitt MC, Zhang Y, Javaid MK, et al. High systemic bone mineral density increases the risk of incident knee $\mathrm{OA}$ and joint space narrowing, but not radiographic progression of existing knee OA: the MOST study. Ann Rheum Dis. 2010; 69: 163-8.

24. Kim YH, Lee JS, Park JH. Association between bone mineral density and knee osteoarthritis in Koreans: the Fourth and Fifth Korea National Health and Nutrition Examination Surveys. Osteoarthr Cartil. 2018 [epub ahead of print].

25. Kellgren JH, Lawrence JS. Radiological assessment of osteo-arthrosis. Ann Rheum Dis. 1957; 16: 494-502.

26. Iwano T, Kurosawa H, Tokuyama H, Hoshikawa Y. Roentgenographic and clinical findings of patellofemoral osteoarthrosis. With special reference to its relationship to femorotibial osteoarthrosis and etiologic factors. Clin Orthop Relat Res. 1990; 252: 190-7.

27. Rosenberg TD, Paulos LE, Parker RD, et al. The forty-five-degree posteroanterior flexion weight-bearing radiograph of the knee. J Bone Joint Surg Am. 1988; 70: 1479-83.

28. van den Driest JJ, Schiphof D, Luijsterburg PAJ, et al. Effectiveness and cost-effectiveness of duloxetine added to usual care for patients with chronic pain due to hip or knee osteo- arthritis: protocol of a pragmatic open-label cluster randomised trial (the DUO trial). BMJ Open. 2017; 7: e018661.

29. Clauw DJ, Hassett AL. The role of centralised pain in osteoarthritis. Clin Exp Rheumatol. 2017; 35: 79-84.

30. Bruyère $\mathrm{O}$, Cooper $\mathrm{C}$, Pelletier J-P, et al. An algorithm recommendation for the management of knee osteoarthritis in Europe and internationally: a report from a task force of the European Society for Clinical and Economic Aspects of Osteoporosis and Osteoarthritis (ESCEO). Semin Arthritis Rheum. 2014; 44: 253-63.

31. Mackenzie IS, Coughtrie MWH, MacDonald TM, Wei L. Antiplatelet drug interactions. J Intern Med. 2010; 268: 516-29.

32. Hsu C-C, Wang H, Hsu Y-H, et al. Use of nonsteroidal anti-inflammatory drugs and risk of chronic kidney disease in subjects with hypertension: Nationwide Longitudinal Cohort Study. Hypertension. 2015; 66: 524-33.

33. Angiolillo DJ, Weisman SM. Clinical pharmacology and cardiovascular safety of naproxen. Am J Cardiovasc Drugs. 2017; 17: 97-107.

34. Tacconelli S, Bruno A, Grande R, et al. Nonsteroidal anti-inflammatory drugs and cardiovascular safety - translating pharmacological data into clinical readouts. Expert Opin Drug Saf. 2017; 16: 791-807.

35. Persson MSM, Fu Y, Bhattacharya A, et al. Relative efficacy of topical non-steroidal antiinflammatory drugs and topical capsaicin in osteoarthritis: protocol for an individual patient data meta-analysis. Syst Rev. 2016; 5: 165.

36. Paoloni M, Bernetti A, Belelli A, et al. Appropriateness of clinical and organizational criteria for intra-articular injection therapies in osteoarthritis. A Delphi method consensus initiative among experts in Italy. Ann Ist Super Sanita. 2015; 51: 131-8.

37. Fioravanti A, Karagulle M, Bender T. Balneotherapy in osteoarthritis: Facts, fiction and gaps in knowledge. EUJIM. 2017; 9: 148-50

38. Verhagen AP, Cardoso JR, Bierma-Zeinstra SMA. Aquatic exercise and balneotherapy in musculoskeletal conditions. Best Pract Res Clin Rheumatol. 2012; 26: 335-43.

39. Beasley J, Ward L, Knipper-Fisher K, et al. Conservative therapeutic interventions for osteoarthritic finger joints: A systematic review. J Hand Ther. 2018 [epub ahead of print].

40. Kloppenburg M, Kroon FP, Blanco FJ, et al. 2018 update of the EULAR recommendations for the management of hand osteoarthritis. Ann Rheum Dis. 2018 [epub ahead of print]. 